Da ultimo pel valore di $\Pi^{2}\left(z_{1}, z_{2} \ldots z_{5}\right)$ trovato nell'articolo suddelto si avrà :

$$
q_{5}=-2 k^{2} k^{\prime 2}\left(1-2 k^{2}\right)
$$

per cui l'equazione di cui le radici sono le $y_{1}, y_{2} \ldots$ sarà la (1).

$\grave{E}$ assai rimarchevole la relazione che ha luogo fra l'equazioni (1) e quella data dal Sigg. Hermite nel suo lavoro "Sur la résolution de l'équation du cinquième degré ” (Comptes Rendus - Mars 1858) Ponendo $y=\mathrm{X} \sqrt[4]{-5 k^{2} k^{12}}$ nella (1) si otliene :

$$
\mathrm{X}^{5}-\mathrm{X}+\frac{2}{5 \sqrt[4]{5}} \frac{1-2 k^{2}}{\sqrt[4]{-k^{2} k^{12}}}=0
$$

e mutando in questa la $k$ in $\frac{1}{k^{\prime}}$ si giunge alla :

$$
\mathrm{X}^{5}-\mathrm{X}-\frac{2}{5 \sqrt[4]{5}} \frac{1+k^{2}}{k \sqrt{ } k}=0
$$

la quale è l'eruazione del Sig. Hermite.

Seltembre 1858.

Prof. F. Brioscul.

\title{
PUBBLICAZIONI RECENTI
}

Dienger - Abbildung krummer Oberfächen auf einander - Braunschtveig. 1858.

Hinsr - - On equally attracting Bodies - Philosophical Magazinc - September 18 s.

Conbescure - Thèse d'Analyse - Sur la théorie analytique des formes homogìnes. Paris 1 Sós.

HEGER - Über die Auflösung eines Systemes von mehreren unbestimmten Gleichungen - Denlaschriften der Akademie der Wissenschaften - Vien - 1858.

Sterver - Vermischte Sätze und Aufgaben - Monatsbericht der Akademie zu Berlin - Juli 18 ä8.

Bierens de HaAn --Raccolta d'Integralt inseríta nelle Memorie de la Società di Amsterdam: il Sig. Bertrand nei Comptes Rendus du 13 Settembre $1858 \mathrm{fa}$ osservare che in quest'importante raccolta vi si trovano riuniti più di ottomila integrali, dei quali più di due mila ne sono stati calcolati dal dotto geometra di Amsterdam.

ERRATA-CORRIGE PEL N: 3:

Pag. 166 linea vitima inveee di $\omega \omega_{1} \omega_{2}$

, $167 \quad 13 \quad \theta^{3} \mathrm{D}=y$

? 14 cubica

, 168 quartulta. 14 terzulta.

sotto

$\mathrm{AD}^{2}+4 \mathrm{C}^{3}$

ovvero

$\begin{array}{cl}\text { leggasi } & \omega \omega_{1} \omega_{3} \\ : & -\theta^{3} \mathrm{D}=y \\ & \text { cubica gobba } \\ & \text { retta } \\ \text { AD } & \text { ottiensi }\end{array}$

in tutta la pag. 171 e nella linea 2 della pag. 172 si mutino $i$ segni di $y$ e $\mu$.

a pag. 172 linea 5 leggasi : $\left\{\left(\theta-\theta_{1}\right)\left(\theta x+\theta_{1} y\right)-\theta \theta_{1} z\right\}^{2}+48 \theta_{1}\left(\theta-\theta_{1}\right)^{2} x y=0$

ERRATA-CORRIGE PEL N: 4:

\section{Errori}

Pag. 206 lin. 27 curva

3213,12 ove

- 222,13 anche

- 225, 10 diventano

, 253. , $2 \sum\left(2 \beta_{l, 1} \beta_{2,2} \ldots \beta_{n, n}\right)$

\section{Correzioni}

carena

ora

ambi

dinotano

$\boldsymbol{\Sigma}\left( \pm b_{1,1} \quad b_{2,2} \ldots b_{n, n}\right)$ 